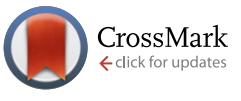

Cite this: Med. Chem. Commun., 2016, 7, 873

Received 6th January 2016, Accepted 15th February 2016

DOI: $10.1039 / \mathrm{c} 6 \mathrm{md} 00004 \mathrm{e}$

www.rsc.org/medchemcomm

\title{
Development and application of ligand-based NMR screening assays for $\gamma$-butyrobetaine hydroxylase $\dagger+$
}

\author{
A. Khan,§ R. K. Leśniak,§ J. Brem, A. M. Rydzik, H. Choi, I. K. H. Leung, đ \\ M. A. McDonough, C. J. Schofield* and T. D. W. Claridge*
}

\begin{abstract}
$\gamma$-Butyrobetaine hydroxylase (BBOX) is a 2-oxoglutarate (2OG) dependent oxygenase that catalyses the stereoselective C-3 hydroxylation of $\gamma$-butyrobetaine (GBB) to give L-carnitine. L-Carnitine is involved in fatty acid metabolism in all animals and in some prokaryotes, and BBOX is a current drug target for the treatment of myocardial infarction. We describe the development and application of ${ }^{1} \mathrm{H} N M R$ GBB/2OG reporter based assays employing paramagnetic relaxation enhancement to monitor inhibitor binding to the BBOX active site. In a single experiment, the method assesses inhibitors for competitive binding with 2OG or GBB, or both. The method was exemplified with a set of isoquinoline-based inhibitors; the results reveal structure-activity relationships that were not predicted from crystallographic studies, with some inhibitors competing 2OG only and some competing both 2OG and GBB. The method will also be applicable to work on the inhibition of other 2OG oxygenases.
\end{abstract}

\section{Introduction}

Ferrous ion $\left(\mathrm{Fe}^{\mathrm{II}}\right.$ ) and 2-oxoglutarate (2OG) dependent oxygenases play multiple biologically important roles in humans and other animals, including in lipid metabolism. ${ }^{1,2}$ $2 \mathrm{OG}$ oxygenases play a central role in the metabolism of the chlorophyll metabolite phytanic acid ${ }^{1}$ and in the biosynthesis of $\mathrm{L}$-carnitine, ${ }^{1,2}$ which is required for fatty acid transport into mitochondria. ${ }^{3}$ Two steps in $\mathrm{L}$-carnitine biosynthesis are catalysed by $20 \mathrm{O}$ oxygenases, i.e. the $\mathrm{C}-3$ hydroxylation of trimethyllysine (TML) and of $\gamma$-butyrobetaine (GBB), which are catalysed by trimethyllysine hydroxylase (TMLH) and $\gamma$-butyrobetaine hydroxylase (BBOX), respectively (Scheme 1). ${ }^{3}$ BBOX (and, maybe, TMLH) are inhibited by mildronate (THP or Met88), ${ }^{4}$ which is given to patients after myocardial infarction to suppress fatty acid metabolism. ${ }^{5}$ As shown by NMR and MS studies, mildronate is a competitive BBOX substrate, undergoing oxidation and subsequent degradation including via a Stevens type rearrangement; ${ }^{6}$ it may also inhibit uptake of dietary L-carnitine. There is, therefore, interest in the

Department of Chemistry, Chemistry Research Laboratory, University of Oxford, 12 Mansfield Road, Oxford, OX1 3TA, UK.

E-mail: christopher.schofield@chem.ox.ac.uk,tim.claridge@chem.ox.ac.uk; Fax: +44 (0)1865 285002; Tel: +44 (0)1865 275658

$\dagger$ Electronic supplementary information (ESI) available. See DOI: 10.1039/ c6md00004e

$\$$ The authors declare no competing interests.

$\S$ These authors contributed equally to this work.

I Current address: School of Chemical Sciences, The University of Auckland, Private Bag 92019, Auckland 1142, New Zealand. development of other types of BBOX inhibitors, for example compounds acting as reversibly binding inhibitors that are competitive with $2 \mathrm{OG}$ and/or GBB, but which are not substrates.

We are interested in developing solution-based NMR methods to complement the extensive crystallographic studies on $20 \mathrm{OG}$ oxygenases, in order to help enable the development of clinically useful inhibitors. ${ }^{2}$ Work using NMR spectroscopy on the $20 \mathrm{G}$ dependent hypoxia inducible factor (HIF) prolyl hydroxylase PHD2, has shown that crystallographically observed inhibitor binding modes do not always reflect those occurring in solutions ${ }^{7}$ and that inhibitors which might be expected to compete with substrates do not always do so. ${ }^{7-9}$

Direct ligand observation, monitoring the attenuation or broadening of ligand NMR resonances in the presence of a target protein, can be an efficient method for analysing the
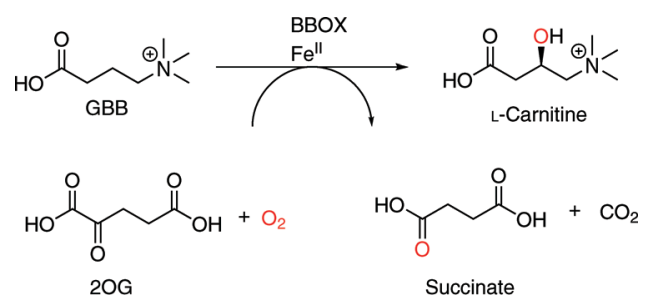

Scheme 1 BBOX catalyses the stereoselective C-3 hydroxylation of GBB to give L-carnitine. The co-substrates are 2OG and oxygen and the co-products are succinate and $\mathrm{CO}_{2}$. 
binding of ligands to a protein and may employ conventional ${ }^{1} \mathrm{H} \mathrm{NMR}^{10-12}$ and/or transverse relaxation-edited ${ }^{1} \mathrm{H} \mathrm{NMR} .{ }^{13,14}$ Site specific ligand binding may also be assessed through ligand competition, i.e. by monitoring the sharpening and recovery of the NMR resonances of displaced ligands, often referred to as spy or reporter molecules. ${ }^{15}$

We describe the development of an NMR assay for recombinant bacterial BBOX from Pseudomonas $s p$. AK1 (psBBOX) ${ }^{16}$ that simultaneously uses both $2 \mathrm{OG}$ and GBB as reporter molecules. We validate the method using a set of isoquinolinebased derivatives, which are established inhibitors of $2 \mathrm{OG}$ oxygenases, including human BBOX (hBBOX). ${ }^{2,17}$ The results reveal that even within the same series of $20 \mathrm{O}$ oxygenase inhibitors, unexpected structure-activity relationships can emerge. The results should help in efforts to develop new BBOX inhibitors and to promote the use of NMR spectroscopy in $20 \mathrm{O}$ oxygenase medicinal chemistry. In developing the assay we employed psBBOX rather than hBBOX, since it can be readily prepared on a large scale and is thought to be structurally closely related to hBBOX. ${ }^{16}$

Several crystal structures have been reported for hBBOX, ${ }^{18-20}$ although none for psBBOX. hBBOX dimerises in an unusual manner using its $\mathrm{N}$-terminal zinc binding domain to interact with the oxygenase domain of the other monomer in the dimer. ${ }^{19,20}$ The general active site architecture of BBOX is very similar to those of other $20 \mathrm{O}$ oxygenases with the active site $\mathrm{Fe}^{\mathrm{II}}$ (to which the oxalyl group of $2 \mathrm{OG}$ binds in a bidentate manner) being ligated by three protein residues. ${ }^{2}$ GBB binds via interactions involving both its trimethylammonium $^{21}$ and carboxylate groups (Fig. 1). The trimethylammonium group is located in an aromatic cage formed by the side chains of Tyr-177, Tyr-194, Trp-181, Tyr-

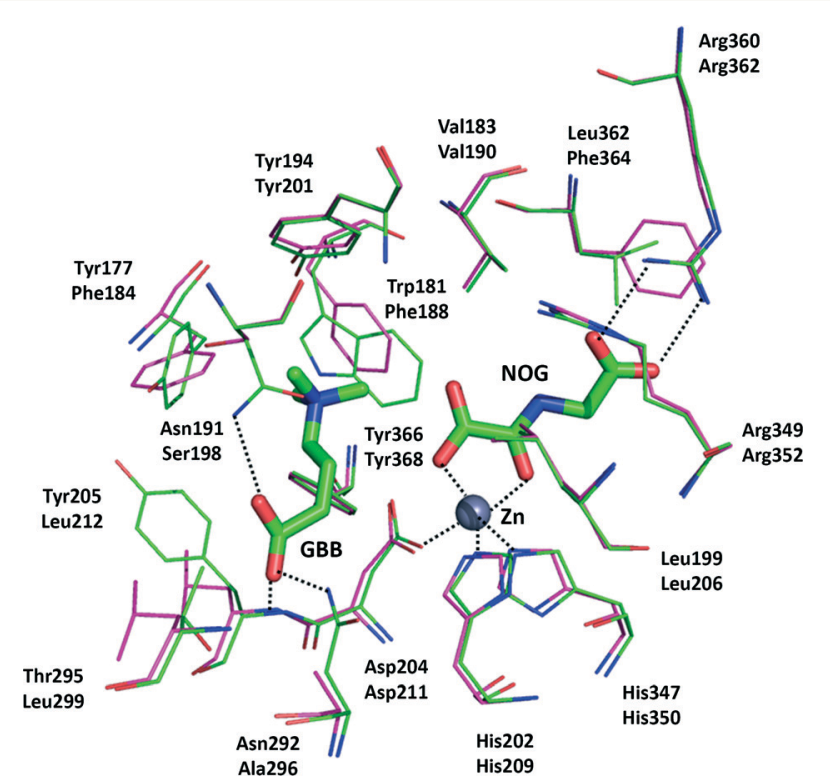

Fig. 1 View from a hBBOX crystal structure (green sticks, upper residues numbers, PDB ID: $302 \mathrm{G})^{20}$ and psBBOX model (pink sticks, lower residue numbers) showing binding of $\mathrm{GBB}$ and $\mathrm{N}$-oxalylglycine (NOG), an unreactive $20 \mathrm{G}$ analogue. $\mathrm{Zn}^{\prime \prime}$ replaces native $\mathrm{Fe}^{\prime \prime}$.
205 and Tyr-366, while the carboxylate group is positioned to interact with the side chains of Asn-292, Asn-191 and the backbone amide of Tyr-205 (Fig. 1).

\section{Results and discussion}

Initially, we optimised the assay conditions to enable detection of binding of both GBB and 2OG (as reporter molecules) to psBBOX in a single Carr-Purcell-Meiboom-Gill (CPMG)edited ${ }^{1} \mathrm{H}$ NMR ligand-observe experiment monitoring line shape changes in the presence of $\operatorname{psBBOX}^{13,14}$ (Fig. 2). The CPMG-edited ${ }^{1} \mathrm{H}$ NMR experiments were used in order to improve NMR sensitivity to ligand binding by enhancing signal attenuation of broadened resonances. To prevent psBBOXcatalysed GBB hydroxylation and uncoupled 2OG decarboxylation into succinate, we replaced the $\mathrm{Fe}^{\mathrm{II}}$ with the 'noncatalytic' metals $\mathrm{Zn}^{\mathrm{II}}$ or $\mathrm{Mn}^{\mathrm{II}}$. We observed that in the presence of psBBOX and $\mathrm{Zn}^{\mathrm{II}}$, the $\mathrm{GBB}$ and $2 \mathrm{OG}$ signals were broadened and attenuated, demonstrating their fastexchange binding interaction with psBBOX (Fig. 2a). However, despite adding high psBBOX concentrations, only relatively moderate broadening was observed. We envisaged that greater sensitivity to ligand binding might be achieved by employing paramagnetic relaxation enhancement (PRE). ${ }^{22,23}$ For this purpose either a paramagnetic metal co-factor such as $\mathrm{Co}^{\mathrm{II}}$ (ref. 24) or $\mathrm{Mn}^{\mathrm{II}}$ (ref. 25, 26) or a protein covalently tagged with a spin label ${ }^{27}$ can be used. When a ligand binds to a protein containing a paramagnetic centre (within $\sim 15$ $\AA$ ), its nuclear spin relaxation rates are enhanced and its NMR signals are significantly broadened. Accordingly, when paramagnetic $\mathrm{Mn}^{\mathrm{II}}$, rather than diamagnetic $\mathrm{Zn}^{\mathrm{II}}$, was added to the BBOX assay, significant increases in the line widths of both GBB and 2OG were observed in the presence of substantially lower amounts of psBBOX as compared to the $\mathrm{Zn}^{\mathrm{II}}$ assay (Fig. 2b). Control experiments (without psBBOX) revealed that at the concentrations employed, free $\mathrm{Mn}^{\mathrm{II}}$ in solution did not affect the GBB line shape; it did, however, broaden the resonances of $2 \mathrm{OG}$ since this directly ligates $\mathrm{Mn}^{\mathrm{II}}$. Despite this broadening, the resonance intensities $\left({ }^{1} \mathrm{H}\right.$ NMR peak areas) remained similar to those prior to the addition of the metal (Fig. $\mathrm{S} 1 \dagger$ ). In a separate control experiment, when the GBB/ 2OG $/ \mathrm{Mn}^{\mathrm{II}} / \mathrm{psBBOX}$ sample was heated $(373 \mathrm{~K}, 5 \mathrm{~min})$ to cause BBOX denaturation, recovery of the reporter signals was observed (Fig. S2 $\dagger$ ), implying that the enhanced broadening of the reporter signals upon the addition of psBBOX was due to binding to the protein. The binding affinities of GBB for both $\mathrm{Zn}^{\text {II }}$ and $\mathrm{Mn}^{\text {II }}$ forms of BBOX in the presence of $2 \mathrm{OG}$ were similar with $K_{\mathrm{D}}$ values of $5 \pm 1$ (Fig. S3 $\dagger$ ) and 4 $\pm 1 \mu \mathrm{M}$ (Fig. $\mathrm{S} 4 \dagger$ ), respectively, as determined by titrating apo-psBBOX into a solution with constant GBB and metal concentrations. The $\mathrm{Mn}^{\mathrm{II}}$ system was therefore chosen for further screening of inhibitors by the NMR dual-reporter displacement assay. In this, competition from a ligand for the GBB and/or 2OG binding sites could be established through the observed recovery of the reporter ligand 

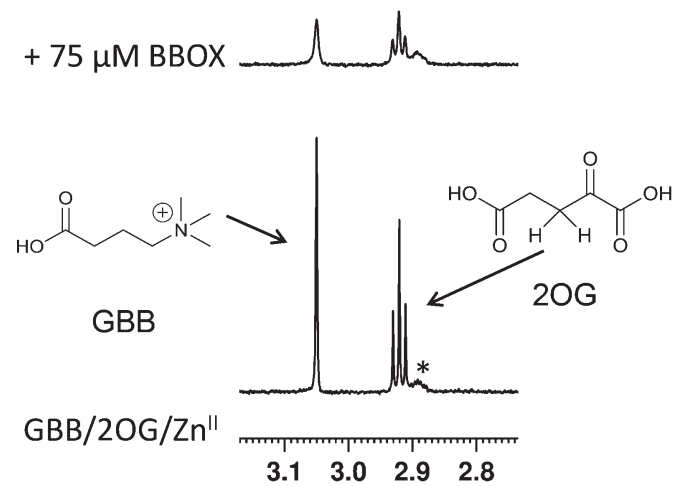

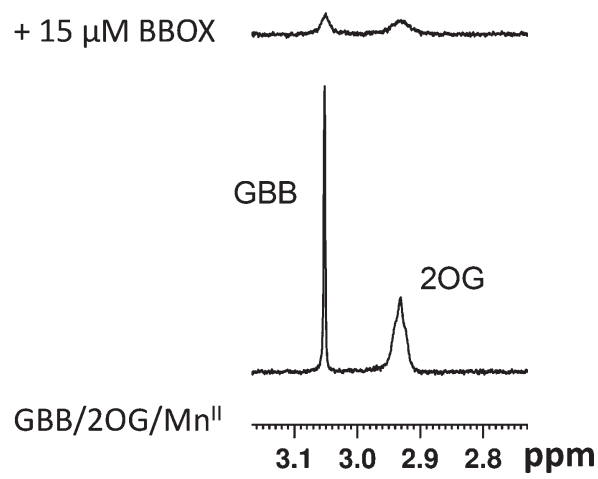

Fig. 2 Monitoring the binding of GBB and 2OG with psBBOX as determined by NMR direct ligand-observation. The GBB (Me 3 singlet at 3.05 ppm) and $2 \mathrm{OG}\left(\mathrm{CH}_{2}\right.$ triplet at $2.91 \mathrm{ppm}$ ) signals from CPMG edited ${ }^{1} \mathrm{H}$ NMR experiments are shown. (a) GBB and $20 \mathrm{OG}$ binding to $\mathrm{BBOX}-\mathrm{Zn}{ }^{11}$ (b) $\mathrm{GBB}$ and $2 O G$ binding to BBOX-Mn". The assay mixture contains $25 \mu \mathrm{M}$ GBB, $300 \mu \mathrm{M} 2 \mathrm{OG}, 150 \mu \mathrm{M} \mathrm{M}$ ", $80 \mathrm{mM}$ KCl, in $50 \mathrm{mM}$ Tris- $\mathrm{D}_{11}$ buffer, pH 7.5 , in $\mathrm{D}_{2} \mathrm{O}$. The asterisk indicates an impurity.

resonances upon addition of the inhibitor to the $\mathrm{GBB} / 2 \mathrm{OG} /$ $\mathrm{Mn}^{\mathrm{II}} / \mathrm{psBBOX}$ sample.

A set of 15 isoquinoline-based compounds ${ }^{17}$ were screened for their binding to psBBOX using simultaneously both GBB and 2OG as reporter molecules (Fig. 3). All these ligands shared the same isoquinoline core skeleton, but differed in their amino-acid derived side chains (Fig. 4). Exemplary results from the assay are presented in Fig. 3 for two of these isoquinoline-based ligands, one possessing the Gly (1) and the other the L-Trp (11) derived side chains. The spectra of GBB and $2 \mathrm{OG}$ with $\mathrm{Mn}^{\mathrm{II}}$ were first recorded without and with psBBOX (Fig. 3a and b), then the isoquinoline-ligand was added to the latter sample. With 1 , only the signals of $2 \mathrm{OG}$ were observed to recover (Fig. 3c), implying that 1 competes efficiently with 2OG, but not GBB. In contrast, with 11, we observed recovery of both $2 \mathrm{OG}$ and GBB signals (Fig. 3d), implying competition with both $2 \mathrm{OG}$ and GBB. The combined results of this assay for all the isoquinoline-ligands tested (at $25 \mu \mathrm{M})$ are summarised in Fig. 4 .

The results imply that the isoquinoline-ligands containing the Gly (1) or Ala (both D, 2 and L, 3) derived side chains compete with 2OG, but not GBB. The full displacement of $20 \mathrm{O}$ by these ligands suggests that they are likely strong binders of psBBOX. The results also imply that binding of these compounds enhanced GBB binding relative to that in the presence of 2OG. On increasing the size of the steric bulk of the side chain, i.e. for those derived from Val $(4,5)$ and Leu $(6$, 7), the ligands appeared to bind less favourably as demonstrated by lower levels, or even a lack, of $20 \mathrm{OG}$ displacement compared to 1, 2 and 3. The apparent displacement of both $2 \mathrm{OG}$ and GBB by 5 and 7, albeit at low levels, suggests that they attenuate GBB binding. Notably, binding was observed only for the L-forms $(5,7)$, but not for the D-forms $(4,6)$, of the Val and Leu derived compounds. This preference for binding by the $\mathrm{L}$-enantiomer was preserved through most of the results where substantial reporter displacement was observed. These results encouraged us to analyse compounds with more hydrophobic or polar side chains (7-15). Ligands with the $\mathrm{D}^{-}$and $\mathrm{L}$-Asp and Glu derived side chains (12-15) were mostly weak $20 \mathrm{O}$ competitors relative to the inhibitors with hydrophobic side chains (5, 7, 9 and 11). 12 and 15 competed weakly with both $2 \mathrm{OG}$ and GBB, whereas, 13 competed with only 2OG, suggesting differences in binding modes, though care should be taken in interpreting results with weakly binding ligands. The results with the more bulky Phe $(8,9)$ and $\operatorname{Trp}(10,11)$ derived side chain ligands were striking. The L-enantiomers 9 and $\mathbf{1 1}$ displaced both $2 \mathrm{OG}$ and GBB clearly contrasting with the ligands with smaller side chains (1-3).

The NMR binding results were then compared with those for the same compounds obtained using an intrinsic fluorescence-based binding assay. ${ }^{17,28}$ The results of fluorescence based assay (Table 1) demonstrate that ligands with Gly 1, D-Ala 2, L-Ala 3, L-Phe 9 and L-Trp 11 are the strongest binders, i.e. they agree with the ${ }^{1} \mathrm{H}$ NMR assay results. They also support the proposal that in general the $\mathrm{L}$-enantiomers bind more tightly than the D-enantiomers. The combined NMR and fluorescence assay results reveal that the level of $2 O G$ displacement correlates with the binding affinity, notably 1, 2, 3, 9 and 11 all fully displace $20 \mathrm{O}$ (within our limits of detection) and have approximately similar binding affinities $\left(K_{\mathrm{D}}=22,20,15,26\right.$ and $29 \mu \mathrm{M}$, respectively). Similarly, ligands 5,7 and 15, all compete relatively moderately with 2OG compared to ligands $1,2,3,9$ and 11 , and have similar binding affinities $\left(K_{\mathrm{D}}=55,55\right.$ and $64 \mu \mathrm{M}$ respectively), which are weaker than 1, 2, 3, 9 and 11. Compounds 12 and 13, which appear to displace $2 \mathrm{OG}$ to a similar extent, also have similar affinities ( $K_{\mathrm{D}}=101$ and $91 \mu \mathrm{M}$ respectively).

We then carried out catalytic turnover assays employing ${ }^{1} \mathrm{H} \mathrm{NMR}^{20}$ (Fig. 5) and fluoride ion release ${ }^{29}$ assays to study 
(d)

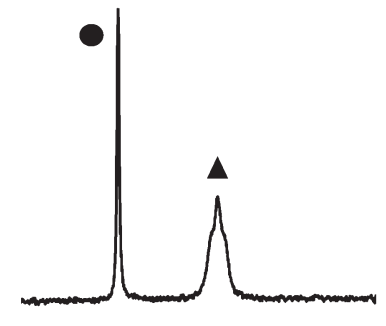

(c)

(b)
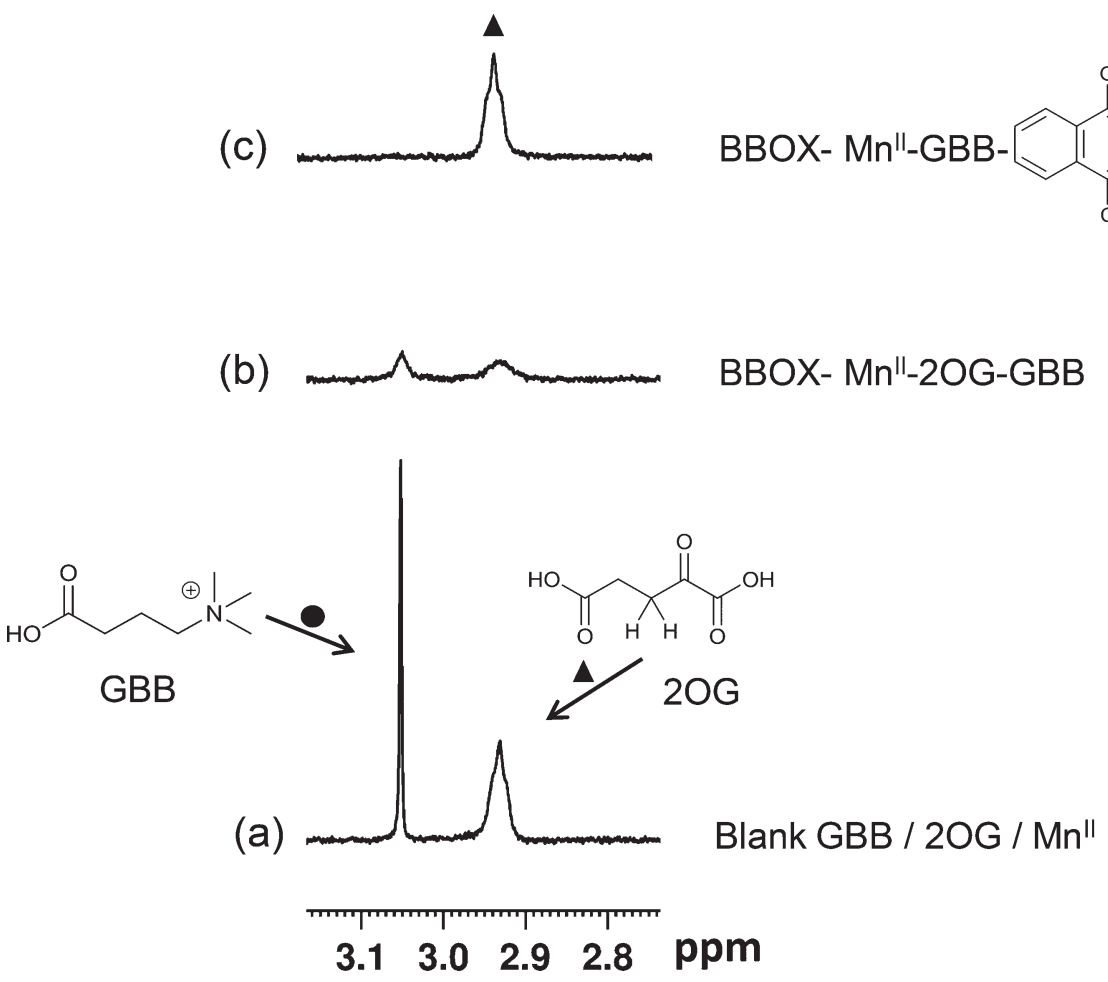

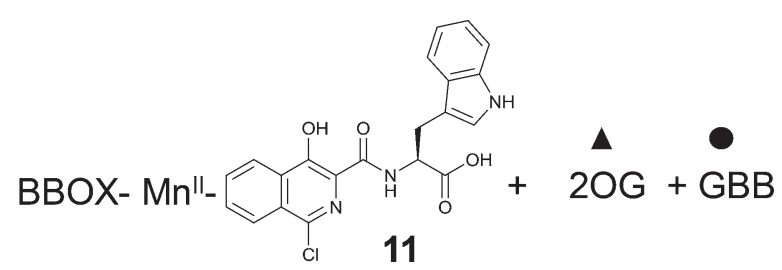

Fig. 3 Studying the binding of isoquinoline ligands 1 and 11 with psBBOX using the NMR dual-reporter displacement assay. 2OG and GBB are used as reporter ligands in CPMG edited ${ }^{1} \mathrm{H}$ NMR experiments. (a) Reporter ligands GBB and 2OG with $\mathrm{Mn}^{11}$ in solution in the absence of psBBOX. (b) After the addition of psBBOX. (c) Addition of 1 to sample of b. (d) Addition of 11 to sample of b. Symbols $(\bullet, \mathbf{\Lambda})$ indicate reporter signal recovery due to displacement. The final assay mixture contained $25 \mu \mathrm{M} \mathrm{GBB}, 300 \mu \mathrm{M} \mathrm{2OG}, 150 \mu \mathrm{M} \mathrm{Mn}$ ", $15 \mu \mathrm{M}$ BBOX, $80 \mathrm{mM}$ KCl and $25 \mu \mathrm{M}$ isoquinoline-based ligand in $50 \mathrm{mM}$ Tris- $\mathrm{D}_{11}$ buffer, $\mathrm{pH} 7.5$, in $\mathrm{D}_{2} \mathrm{O}$.

BBOX inhibition by these isoquinoline-based compounds (Table 1). For the NMR assay, reactions (60 s) were quenched by the addition of $1 \mathrm{M} \mathrm{HCl}$ (Fig. S5†); the concentrations of GBB and L-carnitine were measured by integration of their $\mathrm{Me}_{3}$ resonances (Fig. 5, S5 $\dagger$ ). The fluoride ion release assay ${ }^{29}$ employs BBOX catalysed hydroxylation of (3S)-3-fluoro-4(trimethylammonio)butanoate (GBBF) to give an unstable product which fragments to give a fluoride ion that can subsequently deprotect a tert-butyldimethylsilyl-protected fluorescein to provide measurable fluorescence. Differences in the absolute $\mathrm{IC}_{50}$ values of ${ }^{1} \mathrm{H}$ NMR and fluoride release assays, particularly for weak inhibitors, are likely due to the different experimental conditions used. The fluoride release assay was initially developed for use on hBBOX inhibition; ${ }^{29}$ however, when applied to psBBOX we found the assay required higher concentrations of psBBOX to observe detectable fluorescence when compared to the hBBOX protocol $(1 \mu \mathrm{M} v s .0 .2 \mu \mathrm{M}$, respectively). This concentration difference is notable when considering the amount of enzyme used for the NMR assay $(0.14 \mu \mathrm{M})$. In addition, the NMR assay uses the natural substrate GBB, whereas the fluoride assay necessarily uses GBBF whose affinity for psBBOX is notably different $\left(K_{\mathrm{M}}=2.4 \mathrm{mM}\right.$ and $0.623 \mathrm{mM}$ respectively). ${ }^{16}$ Nonetheless the results from both assays (Table 1) suggest that isoquinoline-based ligands 1, 2, 3, 9 and 11 are the strongest inhibitors in the series, consistent with their ability to compete with 2OG. Similarly, ligands 5, 7 and 15 are medium potency inhibitors, whereas, ligands 4, 6, 8, 10, 12, 13, and 14 are weak inhibitors (Fig. 4, Table 1).

Although we have not yet been able to obtain a BBOX crystal structure with an isoquinoline-based inhibitor, manual docking and energy minimisation studies using a model of psBBOX based on a crystal structure of human BBOX (PDB ID: $3 \mathrm{O} 2 \mathrm{G})^{20}$ suggest a possible structural explanation for the 


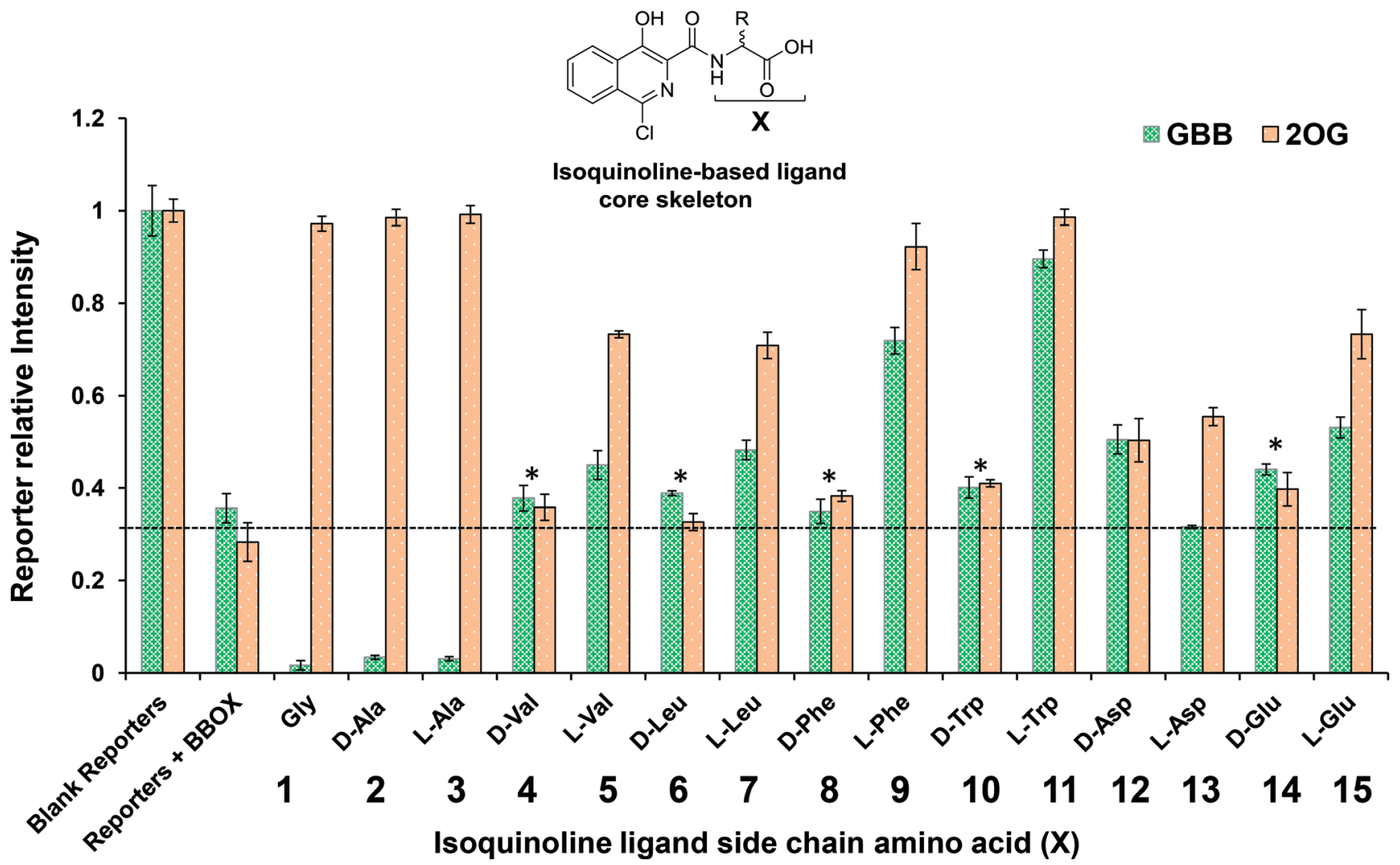

Fig. 4 The chart compares the signal intensity of GBB (green) and 2OG (orange) in the absence of psBBOX (1st left set of columns; intensity set to unit intensity) with their intensity after the addition of psBBOX (2nd left set of columns) and with their intensity after the addition of isoquinolinebased inhibitors 1-15 (side-chain amino acid X varying). The threshold line represents the relative intensity of the reporters in the presence of psBBOX. After the addition of isoquinoline-ligand, the level above this line represents reporter displacement; the level below represents enhancement of reporter binding. Asterisks highlight isoquinoline-ligands whose addition results in a negligible difference on $\mathrm{GBB} / 20 \mathrm{O}$ under these conditions. The final assay mixture contained $25 \mu \mathrm{M}$ GBB, $300 \mu \mathrm{M} 2 \mathrm{OG}, 150 \mu \mathrm{M} \mathrm{Mn}$ ", $15 \mu \mathrm{M} \mathrm{BBOX}, 80 \mathrm{mM} \mathrm{KCl}$ and $25 \mu \mathrm{M}$ isoquinoline-based ligand in $50 \mathrm{mM}$ Tris- $\mathrm{D}_{11}$ buffer, $\mathrm{pH} 7.5$, in $\mathrm{D}_{2} \mathrm{O}$. The error bars represent standard deviations from three separate measurements.

observed trends (Fig. 6). Studies by Rydzik et al. ${ }^{17}$ on hBBOX using the fluoride ion release-based assay have shown that

Table 1 The binding dissociation constant $\left(K_{\mathrm{D}}\right)$ and inhibition constant $\left(\mathrm{IC}_{50}\right)$ values of the isoquinoline-based ligands tested in the study. Fluorescence $K_{\mathrm{D}}$ and ${ }^{1} \mathrm{H}$ NMR IC $\mathrm{C}_{50}$ values are average of triplicates measurements. $F^{-}$release $I_{50}$ values show standard errors for quadruplicate measurements

\begin{tabular}{lllll}
\hline Compound & $\begin{array}{l}\text { Amino } \\
\text { acid } \\
\text { no. }\end{array}$ & $\begin{array}{l}K_{\mathrm{D}}(\mu \mathrm{M}) \text { by } \\
\text { fluorescence } \\
\text { assay }\end{array}$ & $\begin{array}{l}\mathrm{IC}_{50}(\mu \mathrm{M}) \text { by } \\
{ }^{1} \mathrm{H} \text { NMR } \\
\text { assay }\end{array}$ & $\begin{array}{l}\mathrm{IC}_{50}(\mu \mathrm{M}) \text { by } \\
\mathrm{F}^{-} \text {release } \\
\text { assay }\end{array}$ \\
\hline $\mathbf{1}$ & Gly & $22 \pm 4$ & $0.21 \pm 0.01$ & $1.2 \pm 0.1$ \\
$\mathbf{2}$ & D-Ala & $20 \pm 4$ & $0.20 \pm 0.02$ & $0.9 \pm 0.03$ \\
3 & L-Ala & $15 \pm 3$ & $0.22 \pm 0.01$ & $0.7 \pm 0.02$ \\
$\mathbf{4}$ & D-Val & $144 \pm 6$ & $191 \pm 6$ & $>1000$ \\
$\mathbf{5}$ & L-Val & $55 \pm 3$ & $23 \pm 2$ & $130 \pm 14$ \\
$\mathbf{6}$ & D-Leu & $103 \pm 4$ & $94 \pm 8$ & $>1000$ \\
7 & L-Leu & $55 \pm 3$ & $26 \pm 2$ & $125 \pm 6$ \\
$\mathbf{8}$ & D-Phe & $82 \pm 3$ & $38 \pm 0.6$ & $101 \pm 6$ \\
$\mathbf{9}$ & L-Phe & $26 \pm 4$ & $1 \pm 0.03$ & $13 \pm 0.7$ \\
$\mathbf{1 0}$ & D-Trp & $168 \pm 37$ & $18 \pm 1$ & $>1000$ \\
$\mathbf{1 1}$ & L-Trp & $29 \pm 5$ & $0.9 \pm 1$ & $26 \pm 2$ \\
$\mathbf{1 2}$ & D-Asp & $101 \pm 1$ & $38 \pm 1$ & $160 \pm 61$ \\
$\mathbf{1 3}$ & L-Asp & $91 \pm 2$ & $76 \pm 2$ & $245 \pm 62$ \\
$\mathbf{1 4}$ & D-Glu & $89 \pm 6$ & $86 \pm 5$ & $>1000$ \\
$\mathbf{1 5}$ & L-Glu & $64 \pm 2$ & $22 \pm 2$ & $147 \pm 21$
\end{tabular}

for hBBOX, as for psBBOX, the $\mathrm{L}$-configured ligands $\mathbf{3}$ and $\mathbf{1 1}$ are better inhibitors than the corresponding $\mathrm{D}$-forms $(2$ and 10) ( $\mathrm{IC}_{50} 6 \mu \mathrm{M} v s .73 \mu \mathrm{M}$, and $11 \mu \mathrm{M} v s .33 \mu \mathrm{M}$, respectively). The docking results suggest that 1 binds psBBOX possibly by chelating the active site metal via its pyridinium nitrogen and amide carbonyl oxygen, as observed for other $2 \mathrm{OG}$ oxygenases, i.e. PHD2 (PDB ID: 2G1M) ${ }^{30}$ and the fat mass and obesity protein FTO (PDB ID: 4 IE6), ${ }^{31}$ although more than one chelation mode has been observed for related compounds with PHD2. ${ }^{7}$ Removal of the isoquinoline inhibitor pyridinium nitrogen or the hydroxyl group leads to a significant loss in potency against hBBOX. ${ }^{17}$ In our proposed binding mode for 1 , the phenyl ring of the isoquinoline projects towards the GBB binding pocket, causing movement of Phe184, but likely does not disrupt the pocket to the extent that GBB binding is ablated, i.e. it agrees with the NMR observations for 1-3 showing that $20 \mathrm{O}$ but not GBB displacement occurs.

The observation that some ligands with larger hydrophobic side chains $(5,7,9,11)$ compete with both $2 \mathrm{OG}$ and GBB, is interesting. Manual docking of $\mathbf{1 1}$ to the psBBOX model followed by energy minimisation suggests that the bicyclic ring system of these inhibitors is tilted with respect to that of the 

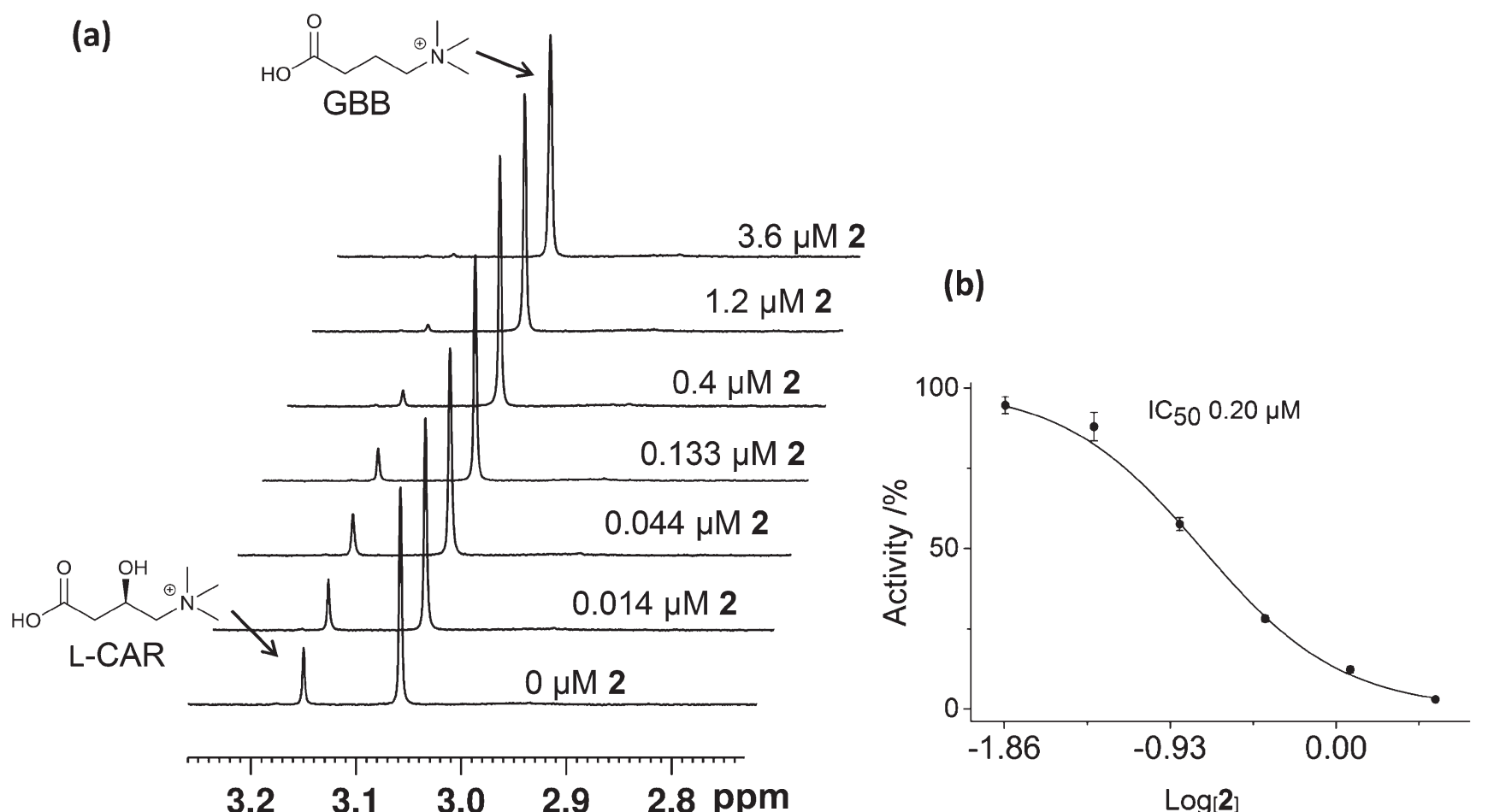

Fig. 5 Measurements of the $I C_{50}$ value of the isoquinoline-ligand 2 by ${ }^{1} \mathrm{H}$ NMR. (a) Bottom to top; psBBOX catalysed GBB turnover into L-carnitine (L-CAR) in the presence of increasing amounts of 2. (b) Dose response curve of (a). Error bars represent standard deviations from three separate measurements. The assay mixture contained $200 \mu \mathrm{M} \mathrm{GBB}, 600 \mu \mathrm{M} 2 \mathrm{OG}, 100 \mu \mathrm{M} \mathrm{Fe",} 500 \mu \mathrm{M}$ L-ascorbate, $80 \mathrm{mM} \mathrm{KCl}$ and $0.140 \mu \mathrm{M} \mathrm{BBOX}$ in 50 $\mathrm{mM}$ Tris- $\mathrm{D}_{11}, \mathrm{pH} 7.5$ in $\mathrm{D}_{2} \mathrm{O}$.

docked/minimised compound 1 while maintaining its metal coordination; this binding mode results in the chlorine and phenyl ring of the inhibitor protruding deeper into the GBB binding site. As a result the 'aromatic cage' residue Phe184

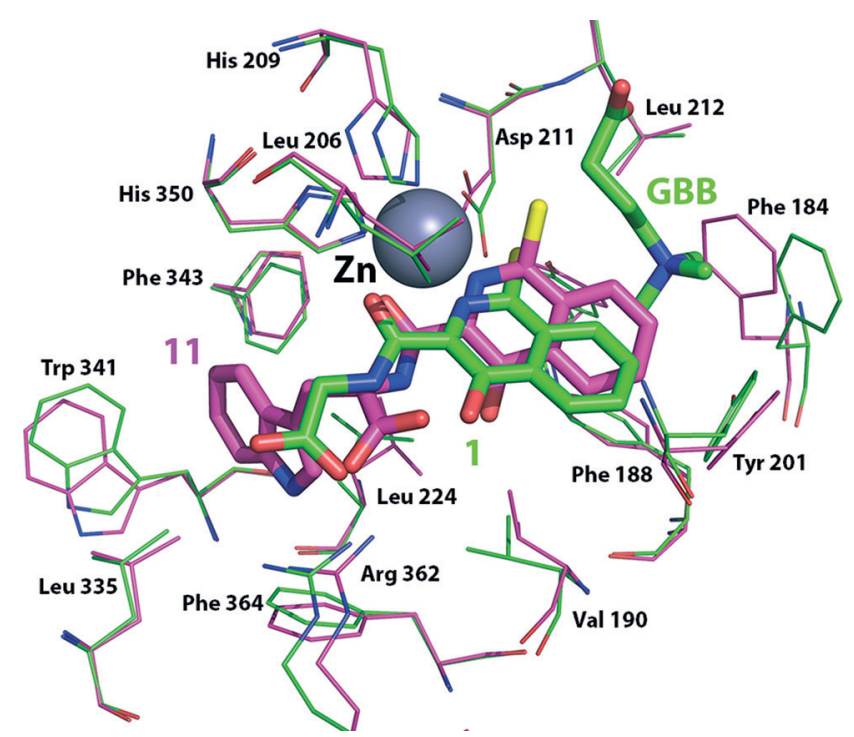

Fig. 6 Docking simulation showing possible conformational changes as a result of binding inhibitors 1 (green sticks, $\mathrm{Cl}$ in yellow) or 11 (pink sticks, $\mathrm{Cl}$ in yellow) to a model of the psBBOX active site based on a hBBOX X-ray crystal structure (PDB ID: $302 \mathrm{G}$ ). ${ }^{20}$ can move approximately $2.5 \AA$ A closer to the aromatic inhibitor thus changing the shape of the aromatic cage and also sterically blocking binding of the GBB trimethylamino group, ${ }^{21}$ consistent with the NMR results, i.e. this inhibitor competes with $2 \mathrm{OG}$ and GBB. Although, alternative binding/chelation modes cannot be ruled out, the modelling studies imply that the larger side chains bind in a largely hydrophobic pocket adjacent to the $20 \mathrm{OG}$ pocket (defined by the side chains of Val-190, Ala-200, Leu-206, Ser-236, Leu-224, Leu-335, Trp-341, Phe-343, Arg-352 and Phe-364); they also imply that binding in this pocket prefers the $\mathrm{L}^{-}$, rather than the $\mathrm{D}^{-}$, stereochemistry as observed (Table 1, Fig. 6) due to a potential clash between the carboxylate of 10 (D-stereochemistry) and Leu-206. It is notable that a loop (residues 190-205) involved in GBB binding also forms part of the hydrophobic pocket and biophysical studies have revealed the occurrence of conformational changes in BBOX catalysis. ${ }^{17}$ The binding of inhibitor 11 may thus elicit changes in the behaviour of this loop and so hinder GBB binding.

\section{Conclusions}

We have developed an efficient ligand-based ${ }^{1} \mathrm{H}$ NMR competitive binding assay for determining inhibitor binding to psBBOX that is operationally simple and requires amounts of materials comparable to other biophysical techniques. The method readily reveals whether an inhibitor disrupts the 
binding of the co-substrate 2OG, the substrate GBB, or both. We envisage the assay will aid in the development of new BBOX inhibitors, including those selective for BBOX over other 2OG oxygenases. More generally, the results show how a readily applied NMR-based method can reveal mechanistic and structural insights not readily determined either by crystallography or by classical kinetic analyses.

\section{Experimental}

\section{Materials}

Chemicals were purchased from Sigma Aldrich, Alfa Aesar and Cortecnet unless otherwise stated. Details of the synthesis of the isoquinoline derivatives have been previously reported..$^{17,32}$

\section{Production and purification of psBBOX AK1}

Recombinant psBBOX AK1 was produced and purified according to the protocol of Rydzik et al. ${ }^{16}$

\section{NMR Experiments}

All NMR experiments were performed at a temperature of $298 \mathrm{~K}$ using a Bruker Avance III $700 \mathrm{MHz}$ spectrometer equipped with a TCI inverse cryoprobe or a Bruker Avance III $600 \mathrm{MHz}$ spectrometer equipped with a $5 \mathrm{~mm} \mathrm{BB}-\mathrm{F} /{ }^{1} \mathrm{H}$ Prodigy $\mathrm{N}_{2}$ cryoprobe. The PROJECT - CPMG pulse sequence $\left(90^{\circ}{ }_{x}-\left[\tau-180^{\circ}{ }_{y}-\tau-90^{\circ} y-\tau-180^{\circ} y-\tau\right]_{n}-\text { acq }\right)^{33}$ was used to attenuate broad resonances, using a total echo time of $32 \mathrm{~ms}$. All spectra were processed with a Lorentzian line broadening of $0.3 \mathrm{~Hz}$ and were referenced to an internal standard (1,1,1 trifluoroacetone at $1.49 \mathrm{ppm})$. BBOX titration data for measuring the $K_{\mathrm{D}}$ of GBB were fitted using OriginPro 9.0 (Origin lab, Northampton, MA, USA). Bruker MATCH (3 $\mathrm{mm}$ diameter) and $5 \mathrm{~mm}$ NMR tubes with a total sample volume of $160 \mu \mathrm{L}$ and $500 \mu \mathrm{L}$, respectively, were used. The solutions were buffered in $50 \mathrm{mM}$ Tris- $\mathrm{D}_{11} \cdot \mathrm{DCl}$, $\mathrm{pH} 7.5$, in $\mathrm{D}_{2} \mathrm{O}$. For each sample the pulse tip-angle calibration was carried out using the single-pulse nutation method. ${ }^{34}$ Each experiment was recorded with three separately prepared samples.

\section{NMR IC so $_{\text {measurements }}$}

The GBB turnover to L-carnitine was initiated by the addition of psBBOX to the assay mixture in a $1.5 \mathrm{~mL}$ plastic Eppendorf tube at ambient temperature (294 K), and was quenched after $60 \mathrm{~s}$ by addition of $10 \mu \mathrm{l} 1 \mathrm{M} \mathrm{HCl}$. The assay mixture contained $200 \mu \mathrm{M}$ GBB, $600 \mu \mathrm{M}$ 2OG, $100 \mu \mathrm{M} \mathrm{Fe}$ (from $\mathrm{Fe}\left(\mathrm{NH}_{4}\right)_{2}\left(\mathrm{SO}_{4}\right)_{2}$ salt, which was prepared as a $250 \mathrm{mM}$ stock solution in $20 \mathrm{mM} \mathrm{HCl}$ which was further diluted to a 2.5 $\mathrm{mM}$ solution in MilliQ purified water), $500 \mu \mathrm{M}$ L-ascorbate, and $80 \mathrm{mM} \mathrm{KCl}$ in $50 \mathrm{mM}$ Tris- $\mathrm{D}_{11}, \mathrm{pH} 7.5$ in $\mathrm{D}_{2} \mathrm{O}$, leading to a final psBBOX concentration of $0.14 \mu \mathrm{M}$. The psBBOX activity (\%) at a given inhibitor concentration was measured as $\left([\mathrm{CAR}]_{\mathrm{P}+\mathrm{I}} /[\mathrm{CAR}]_{\mathrm{P}}\right) \times 100$, where $\mathrm{P}=$ protein, $\mathrm{I}=$ inhibitor.
Response curves were fitted using OriginPro 9.0 (Origin lab, Northampton, MA, USA).

Binding assay $\left(K_{\mathrm{D}}\right)$ - intrinsic fluorescence quenching ${ }^{17,28}$

$K_{\mathrm{D}}$ values were determined from the quenching of intrinsic tryptophan fluorescence measured at $294 \mathrm{~K}$ using a Pherastar FS plate reader (BMG labtech) with 96-well plates (Greiner, black, bottom: flat, clear). The following conditions were used: excitation $280 \mathrm{~nm}$, emission $350 \mathrm{~nm}, 5 \mu \mathrm{M}$ psBBOX, $50 \mu \mathrm{M} \mathrm{Mn}{ }^{\mathrm{II}}$ and varying concentrations of inhibitors to a final well volume of $50 \mu \mathrm{L}$ in $50 \mathrm{mM}$ Tris buffer $\mathrm{pH} 7.5$, supplemented with $200 \mathrm{mM} \mathrm{NaCl}$. Fluorescence readings were obtained in triplicate with errors represented as standard deviations. $K_{\mathrm{D}}$ values were obtained from the raw data by plotting inhibitor concentration against $\Delta F_{\text {obs }}$ l $F_{\text {max }}\left(\Delta F_{\text {obs }}\right.$ is the decrease in observed fluorescence and $F_{\max }$ is the observed fluorescence signal without any inhibitor present).

\section{Fluoride release assay $\left(\mathrm{IC}_{50}\right)$}

$\mathrm{IC}_{50}$ measurements were obtained using a fluoride release assay, as previously applied to hBBOX. ${ }^{29}$ The fluorescence signal was detected using a Pherastar FS plate reader (BMG labtech) fitted with a FITC FP 485/30 (485 nm, bandwidth 30 nm) and FITC FP 520/40 emission (520 nm, bandwidth 40 nm) filters, using 384-well plates (black, clear, flat bottom, Grenier BioOne). GBBF and the TBS-protected fluorescein probe were synthesised according to the published protocol. ${ }^{29}$ The following conditions were used: $50 \mu \mathrm{M}$ GBBF, 500 $\mu \mathrm{M}$ 2OG, $250 \mu \mathrm{M}$ ascorbate, varying concentrations of inhibitors, $50 \mu \mathrm{M} \mathrm{Fe}$ (from $\mathrm{Fe}\left(\mathrm{NH}_{4}\right)_{2}\left(\mathrm{SO}_{4}\right)_{2}$ salt, which was prepared as a $100 \mathrm{mM}$ stock solution in $20 \mathrm{mM} \mathrm{HCl}$ which was further diluted to a $250 \mu \mathrm{M}$ solution in MilliQ purified water before being added at the start of the measurements). Initiation of the assay was carried out by addition of psBBOX to each well containing all inhibitors and cofactors, to a concentration of $1 \mu \mathrm{M}$. Reactions were carried out in a final well volume of $10 \mu \mathrm{L}$ in $50 \mathrm{mM}$ Tris buffer $\mathrm{pH} 7.5$ at $294 \mathrm{~K}$, supplemented with $200 \mathrm{mM} \mathrm{NaCl}$. After 10 minutes, the reaction was quenched by addition of $40 \mu \mathrm{L}$ of TBS-protected fluorescein probe (final concentration $5 \mu \mathrm{M}$ ). The plates were then sealed and incubated for 1 hour at room temperature. Following this, $10 \mu \mathrm{L}$ of HEPES buffer $\mathrm{pH} 7.0$ was added and the fluorescence signal read up to 5 minutes after the addition of HEPES. Fluorescence data were recorded as quadruplicates and errors represented as standard deviations. Fluorescence signals were normalized by subtracting fluorescence values obtained from control wells which contained all reagents, but without enzyme or without inhibitor. $\mathrm{IC}_{50} \mathrm{~S}$ were obtained from the raw data using the Prism four parameter logistic dose-response model (sigmoidal, variable slope).

\section{Acknowledgements}

We thank the following for financial support: Abdul Wali Khan University, Mardan (AWKUM) Pakistan (A. K.), Biotechnology and Biological Sciences Research Council (BBSRC), the 
British Heart Foundation, Wellcome Trust and Cancer Research UK.

\section{References}

1 2-Oxoglutarate-Dependent Oxygenases, ed. R. P. Hausinger and C. J. Schofield, Royal Society of Chemistry, Cambridge, 2015.

2 N. R. Rose, M. A. McDonough, O. N. King, A. Kawamura and C. J. Schofield, Chem. Soc. Rev., 2011, 40, 4364-4397.

3 F. M. Vaz and R. J. Wanders, Biochem. J., 2002, 361, 417-429.

4 B. Z. Simkhovich, Z. V. Shutenko, D. V. Meirena, K. B. Khagi, R. J. Mezapuke, T. N. Molodchina, I. J. Kalvins and E. Lukevics, Biochem. Pharmacol., 1988, 37, 195-202.

5 M. Dambrova, E. Liepinsh and I. Kalvinsh, Trends Cardiovasc. Med., 2002, 12, 275-279.

6 L. Henry, I. K. H. Leung, T. D. W. Claridge and C. J. Schofield, Bioorg. Med. Chem. Lett., 2012, 22, 4975-4978.

7 L. Poppe, C. M. Tegley, V. Li, J. Lewis, J. Zondlo, E. Yang, R. J. Kurzeja and R. Syed, J. Am. Chem. Soc., 2009, 131, 16654-16655.

8 M. C. Chan, O. Atasoylu, E. Hodson, A. Tumber, I. K. H. Leung, R. Chowdhury, V. Gomez-Perez, M. Demetriades, A. M. Rydzik, J. Holt-Martyn, Y. M. Tian, T. Bishop, T. D. W. Claridge, A. Kawamura, C. W. Pugh, P. J. Ratcliffe and C. J. Schofield, PLoS One, 2015, 10, e0132004.

9 I. K. H. Leung, M. Demetriades, A. P. Hardy, C. Lejeune, T. J. Smart, A. Szollossi, A. Kawamura, C. J. Schofield and T. D. W. Claridge, J. Med. Chem., 2013, 56, 547-555.

10 M. D. Shortridge, D. S. Hage, G. S. Harbison and R. Powers, J. Comb. Chem., 2008, 10, 948-958.

11 M. Sarrazin, J. C. Sari, M. Bourdeaux-Pontier and C. Briand, Mol. Pharmacol., 1979, 15, 71-77.

12 J. J. Fischer and O. Jardetzky, J. Am. Chem. Soc., 1965, 87, 3237-3244.

13 C. Dalvit, A. D. Gossert, J. Coutant and M. Piotto, Magn. Reson. Chem., 2011, 49, 199-202.

14 P. J. Hajduk, E. T. Olejniczak and S. W. Fesik, J. Am. Chem. Soc., 1997, 119, 12257-12261.

15 W. Jahnke, P. Floersheim, C. Ostermeier, X. Zhang, R. Hemmig, K. Hurth and D. P. Uzunov, Angew. Chem., Int. Ed., 2002, 41, 3420-3423.

16 A. M. Rydzik, I. K. H. Leung, G. T. Kochan, N. D. Loik, L. Henry, M. A. McDonough, T. D. W. Claridge and C. J. Schofield, Org. Biomol. Chem., 2014, 12, 6354-6358.

17 A. M. Rydzik, R. Chowdhury, G. T. Kochan, S. T. Williams, M. A. McDonough, A. Kawamura and C. J. Schofield, Chem. Sci., 2014, 5, 1765-1771.
18 K. Tars, J. Leitans, A. Kazaks, D. Zelencova, E. Liepinsh, J. Kuka, M. Makrecka, D. Lola, V. Andrianovs, D. Gustina, S. Grinberga, E. Liepinsh, I. Kalvinsh, M. Dambrova, E. Loza and O. Pugovics, J. Med. Chem., 2014, 57, 2213-2236.

19 K. Tars, J. Rumnieks, A. Zeltins, A. Kazaks, S. Kotelovica, A. Leonciks, J. Sharipo, A. Viksna, J. Kuka and E. Liepinsh, Biochem. Biophys. Res. Commun., 2010, 398, 634-639.

20 I. K. H. Leung, T. J. Krojer, G. T. Kochan, L. Henry, F. von Delft, T. D. W. Claridge, U. Oppermann, M. A. McDonough and C. J. Schofield, Chem. Biol., 2010, 17, 1316-1324.

21 J. J. A. G. Kamps, A. Khan, H. Choi, R. K. Lesniak, J. Brem, A. M. Rydzik, M. A. McDonough, C. J. Schofield, T. D. W. Claridge and J. Mecinović, Chem. - Eur. J., 2016, 22, 1270-1276.

22 I. Bertini, C. Luchinat, G. Parigi and R. Pierattelli, ChemBioChem, 2005, 6, 1536-1549.

23 I. Bertini, C. Luchinat and G. Parigi, Solution NMR of paramagnetic molecules: applications to metallobiomolecules and models, Elsevier, Amsterdam, 2001.

24 I. Bertini, M. Fragai, Y. M. Lee, C. Luchinat and B. Terni, Angew. Chem., Int. Ed., 2004, 43, 2254-2256.

25 I. K. H. Leung, E. Flashman, K. K. Yeoh, C. J. Schofield and T. D. W. Claridge, J. Med. Chem., 2010, 53, 867-875.

26 I. Bertini, M. Fragai, C. Luchinat and E. Talluri, Angew. Chem., Int. Ed., 2008, 47, 4533-4537.

27 W. Jahnke, S. Rudisser and M. Zurini, J. Am. Chem. Soc., 2001, 123, 3149-3150.

28 A. M. Rydzik, J. Brem, W. B. Struwe, G. T. Kochan, J. L. Benesch and C. J. Schofield, Bioorg. Med. Chem. Lett., 2014, 24, 4954-4957.

29 A. M. Rydzik, I. K. H. Leung, G. T. Kochan, A. Thalhammer, U. Oppermann, T. D. W. Claridge and C. J. Schofield, ChemBioChem, 2012, 13, 1559-1563.

30 M. A. McDonough, V. Li, E. Flashman, R. Chowdhury, C. Mohr, B. M. Lienard, J. Zondlo, N. J. Oldham, I. J. Clifton, J. Lewis, L. A. McNeill, R. J. Kurzeja, K. S. Hewitson, E. Yang, S. Jordan, R. S. Syed and C. J. Schofield, Proc. Natl. Acad. Sci. U. S. A., 2006, 103, 9814-9819.

31 W. Aik, M. Demetriades, M. K. Hamdan, E. A. Bagg, K. K. Yeoh, C. Lejeune, Z. Zhang, M. A. McDonough and C. J. Schofield, J. Med. Chem., 2013, 56, 3680-3688.

32 S. S. van Berkel, J. Brem, A. M. Rydzik, R. Salimraj, R. Cain, A. Verma, R. J. Owens, C. W. Fishwick, J. Spencer and C. J. Schofield, J. Med. Chem., 2013, 56, 6945-6953.

33 J. A. Aguilar, M. Nilsson, G. Bodenhausen and G. A. Morris, Chem. Commun., 2012, 48, 811-813.

34 P. S. Wu and G. Otting, J. Magn. Reson., 2005, 176, 115-119. 